\title{
Heating of the molecular gas in the massive outflow of the local ultraluminous-infrared and radio-loud galaxy $4 \mathrm{C} 12.50$
}

\author{
K. M. Dasyra ${ }^{1}$, F. Combes ${ }^{1}$, G. S. Novak ${ }^{1}$, M. Bremer ${ }^{2}$, L. Spinoglio ${ }^{3}$, M. Pereira Santaella ${ }^{3}$, \\ P. Salomé ${ }^{1}$, and E. Falgarone ${ }^{1}$
}

\author{
1 Observatoire de Paris, LERMA (UMR 8112), 61 Av. de l'Observatoire, 75014 Paris, France \\ e-mail: kalliopi.dasyra@obspm.fr \\ 2 Institut de Radio Astronomie Millimétrique, 300 rue de la Piscine, 38406 Saint-Martin d'Hères, France \\ ${ }^{3}$ Istituto di Astrofisica e Planetologia Spaziali, INAF-IAPS, via Fosso del Cavaliere 100, 00133 Roma, Italy
}

Received 17 November 2013 / Accepted 13 February 2014

\begin{abstract}
We present a comparison of the molecular gas properties in the outflow vs. in the ambient medium of the local prototype radio-loud and ultraluminous-infrared galaxy 4C12.50 (IRAS 13451+1232), using new data from the IRAM Plateau de Bure Interferometer and $30 \mathrm{~m}$ telescope and from the Herschel space telescope. Previous $\mathrm{H}_{2}(0-0) \mathrm{S}(1)$ and $\mathrm{S}(2)$ observations with the Spitzer space telescope had indicated that the warm $(\sim 400 \mathrm{~K})$ molecular gas in $4 \mathrm{C} 12.50$ is made up of a $1.4( \pm 0.2) \times 10^{8} M_{\odot}$ ambient reservoir and a $5.2( \pm 1.7) \times 10^{7} M_{\odot}$ outflow. The new $\mathrm{CO}(1-0)$ data cube indicates that the corresponding cold $(25 \mathrm{~K}) \mathrm{H}_{2}$ gas mass is $1.0( \pm 0.1) \times$ $10^{10} M_{\odot}$ for the ambient medium and $<1.3 \times 10^{8} M_{\odot}$ for the outflow, when using a CO-intensity-to- $\mathrm{H}_{2}$-mass conversion factor $\alpha$ of $0.8 M_{\odot} /\left(\mathrm{K} \mathrm{km} \mathrm{s}^{-1} \mathrm{pc}^{2}\right)$. The combined mass outflow rate is high, 230-800 $M_{\odot} / \mathrm{yr}$, but the amount of gas that could escape the galaxy is low. A potential inflow of gas from a $3.3( \pm 0.3) \times 10^{8} M_{\odot}$ tidal tail could moderate any mass loss. The mass ratio of warmto-cold molecular gas is $\gtrsim 30$ times higher in the outflow than in the ambient medium, indicating that a non-negligible fraction of the accelerated gas is heated to temperatures at which star formation is inefficient. This conclusion is robust against the use of different $\alpha$ factor values and/or different warm gas tracers $\left(\mathrm{H}_{2}\right.$ vs. $\mathrm{H}_{2}$ plus $\mathrm{CO}$ ). With the CO-probed gas mass at least 40 times lower at $400 \mathrm{~K}$ than at $25 \mathrm{~K}$, the total warm-to-cold mass ratio is always lower in the ambient gas than in the entrained gas. Heating of the molecular gas could facilitate the detection of new outflows in distant galaxies by enhancing their emission in intermediate rotational number CO lines.
\end{abstract}

Key words. ISM: jets and outflows - ISM: kinematics and dynamics - line: profiles - galaxies: active - galaxies: nuclei infrared: galaxies

\section{Introduction}

Mass accretion events onto black holes (BHs) can release more energy than the binding energy of gas in galaxies (Fabian 2012), and part of this energy can be deposited onto the interstellar medium (ISM) through radiation, accretion-disk winds, and radio jets. The energy carried by these so-called feedback mechanisms can excite or kinematically distort gas on large scales (e.g., over areas of square kiloparsecs; Lípari et al. 2004; Fu \& Stockton 2009; Rupke \& Veilleux 2011; Westmoquette et al. 2012). It can even affect galaxies outside the active galactic nucleus (AGN) that generated it (e.g., Croft et al. 2006; Cantalupo et al. 2012). The effects of feedback on the evolution of galaxies are most severe if part of the gas is accelerated beyond escape velocity. The high-velocity gas will then be lost to the intergalactic medium. Any star formation associated with it will be quenched. For the remaining gas, star formation will be delayed. The lowvelocity gas will cool radiatively, resettle in a disk, and restart its collapse toward the formation of new cores. For these reasons, AGN feedback has been considered as a source of negative feedback, capable of regulating galaxy growth. Positive feedback, i.e., compression of gas leading to an enhancement of star formation, has also been occasionally suggested (e.g., van Breugel et al. 1985; Gaibler et al. 2012; Silk 2013).

AGN feedback, implemented either as a source of heating coupled to the hot gas or as a source of momentum coupled to the cold gas, was included in cosmological simulations to suppress star formation at earlier epochs and to make the predicted number counts of massive present-day galaxies match the observed ones (Granato et al. 2004; Croton et al. 2006; Bower et al. 2006; Somerville et al. 2008; Booth \& Schaye 2009; Debuhr et al. 2012). The claim that feedback could change the high-mass end of the local galaxy mass function by more than an order of magnitude has however been contested by Birnboim et al. (2007), Dekel \& Birnboim (2008), and Bernardi et al. (2013), who argue that the numbers could be revised downward through, e.g., different stellar population modeling assumptions.

The change in the galaxy mass function by AGN feedback remains to be quantified or constrained by observations. To quantify it, we need to determine the frequency of AGN-driven outflows that can considerably delay or quench star formation in their host galaxies. Even though high-velocity outflows have long been identified in optical and radio data of ionized and neutral atomic gas tracers in local AGN (e.g., Heckman et al. 2000; Rupke et al. 2005; Martin 2005; Morganti et al. 2005; Holt et al. 2008), this research field became particularly appealing thanks to the launch of infrared (IR) space missions (e.g., Herschel) and the improvement on the sensitivity and the spectral range of ground-based $\mathrm{mm}$ facilities, which enabled us to probe the molecular gas content of outflows. As the densest ISM component, the molecular gas can contribute considerably to the mass of the entrained gas, if not dominate it. Motions of molecular 


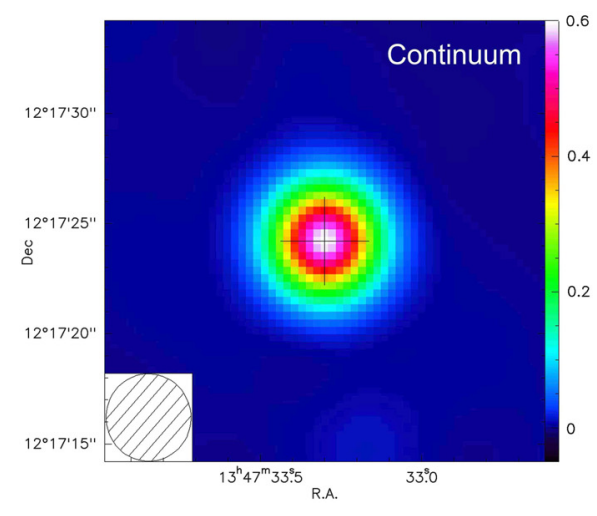

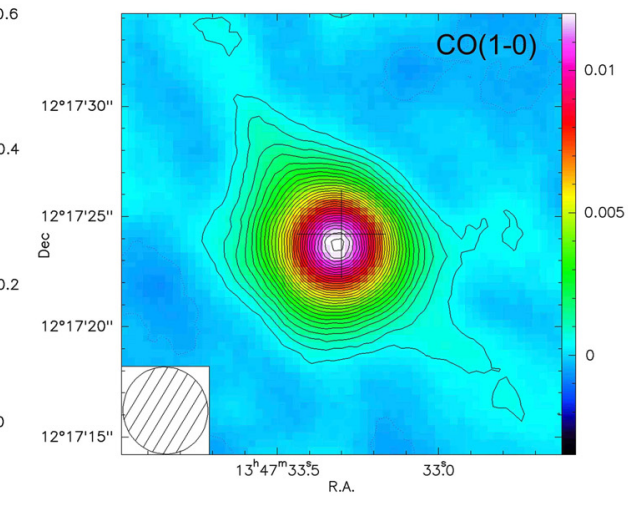

Fig. 1. Left: $102 \mathrm{GHz}$ continuum image of $4 \mathrm{C} 12.50$ (in units of Jy/beam). Right: continuum-free $\mathrm{CO}(1-0)$ line intensity map of $4 \mathrm{C} 12.50$ averaged over the $-500 \mathrm{~km} \mathrm{~s}^{-1}$ to $500 \mathrm{~km} \mathrm{~s}^{-1}$ range, showing extended emission that is offset from the continuum peak (marked with a cross). The contours are at the $3 \sigma$ levels for this image, i.e., in steps of $0.36 \mathrm{mJy} / \mathrm{beam}$. gas that could be associated with a nuclear outflow have been presented for $\sim 50$ local galaxies to date (Sakamoto et al. 2006, 2009; Leon et al. 2007; Feruglio et al. 2010; Fischer et al. 2010; Alatalo et al. 2011; Rangwala et al. 2011; Sturm et al. 2011; Krips et al. 2011; Dasyra \& Combes 2011, 2012; Aalto et al. 2012; Tsai et al. 2012; Morganti et al. 2013a; Combes et al. 2013; Spoon et al. 2013; Veilleux et al. 2013; Cicone et al. 2014). Mass measurements of the accelerated gas were presented in around one fifth of the cases and found to be in the range $10^{6}-10^{9} M_{\odot}$.

To identify spectral lines with strong outflow signatures and use them for the systematic and efficient detection of new outflows, we need to perform excitation studies of the gas in the flow, motivated by the argument that the AGN feedback could leave both kinematic and excitation signatures on the ISM. Such studies have nonetheless only been presented for a couple of sources. In Mrk 231, Cicone et al. (2012) found no evidence of any strong contribution of shocks on the excitation of outflowing $\mathrm{CO}$ molecules at states of low rotational number $J$ : the $\mathrm{CO}(2-1) / \mathrm{CO}(1-0)$ flux ratio was comparable in the outflow and in the ambient medium.

In this paper, we present evidence of heating of the molecular gas in the outflow of the local radio-loud and ultraluminousinfrared system 4C12.50 (IRAS 13451+1232), i.e., a late-stage merger of two galaxies where the nuclei are separated by $4.4 \mathrm{kpc}$ and surrounded by smaller structures (Batcheldor et al. 2007). A relativistic radio jet with an intrinsic bulk flow speed of at least $0.8 c$ emerges from the system's west nucleus (Lister et al. 2003). The setup of a radio jet propagating through a gasrich galaxy, which is rare in the local Universe but common at high redshift (e.g., Sajina et al. 2008; Ivison et al. 2012), makes 4C12.50 an excellent target for studying the properties of the outflowing molecular gas. Moreover, 4C12.50 was the only source in the Spitzer archive for which a warm molecular gas outflow was discovered and characterized. An outflow-related wing was detected in two purely rotational $\mathrm{H}_{2}$ lines, providing the entrained gas excitation temperature and mass (Dasyra \& Combes 2011). Cold gas was also discovered in the outflow of $4 \mathrm{C} 12.50$ thanks to absorption seen in $\mathrm{CO}(2-3)$ with a significant optical deph against the millimeter continuum source (Dasyra \& Combes 2012). In this paper, we compare the properties of the gas in the outflow vs. in the ambient medium using new submillimeter and millimeter data, and we discuss the implications of our findings for future searches of massive molecular gas outflows. Throughout our work, we adopt a $\Lambda$ CDM cosmology with $H_{0}=70 \mathrm{~km} \mathrm{~s}^{-1} \mathrm{Mpc}^{-1}, \Omega_{\mathrm{M}}=0.3$, and $\Omega_{\Lambda}=0.7$.

\section{Data}

The CO(1-0) observations were carried out with the Plateau de Bure (PdB) Interferometer of the Institut de Radioastronomie
Millimétrique (IRAM) as part of the program V028 (PI Dasyra). The data were taken on four different days between September 2011 and January 2012, during which four, five, or six antennae were placed in compact $(\mathrm{C}, \mathrm{D}$, or special) configurations. The dual polarization SIS receivers were tuned at a frequency $v$ of $102.761 \mathrm{GHz}$, and used with the WIDEX backend. The typical weather conditions were $0.6-2.2^{\prime \prime}$ of seeing and $2-10 \mathrm{~mm}$ of water vapor. Bandpass calibrators $(0923+392,1354+195,3 C 84$, 3C 279) were observed before our primary target during the first two runs. During the next two runs, they were observed before and after each 22.5 min-long integration on 4C12.50. This change to the observational strategy enabled us to minimize the scatter due to the strong millimeter continuum of $4 \mathrm{C} 12.50$ by monitoring changes in the time-variable bandpass solution and thus increasing the accuracy of the subsequent phase and flux calibrations. To perform the last two tasks, we used the default IRAM environment, CLIC ${ }^{1}$, selecting 4C12.50 itself for the phase calibration and MWC 349 for the flux calibration. The calibrated visibilities of all tracks were merged into a single table, of depth equivalent to $12.5 \mathrm{~h}$ of observations with a six-antenna array. A cube of $0.34^{\prime \prime}$ per spatial pixel was created from this table using the default PdB image reconstruction environment, MAPPING $^{1}$. Its $1 \sigma$ noise is $1.1 \mathrm{mJy}$ per beam at a spectral resolution of $5.7 \mathrm{~km} \mathrm{~s}^{-1}$. The clean $3 \mathrm{~mm}$ beam corresponds to an ellipse with semi-major and semi-minor axis of $4.0^{\prime \prime}$ and 3.8", respectively, at a position angle of $14^{\circ}$. We constructed the continuum image by computing the median of all cube planes in the $450 \mathrm{~km} \mathrm{~s}^{-1}$ to $1250 \mathrm{~km} \mathrm{~s}^{-1}$ range, and removed it from the entire cube to obtain a continuum-free $\mathrm{CO}(1-0)$ line cube (Fig. 1).

Observations at $205.522 \mathrm{GHz}$ targeting the $\mathrm{CO}(2-1)$ line were carried out in June 2012 with the IRAM 30 m telescope. The Eight Mixer Receiver (EMIR; Carter et al. 2012) and the Wideband Line Multiple Autocorrelator (WILMA) backend were used for this program. The FTS backend was also connected for repeatability. The data presented here are rather shallow, at $20 \mathrm{~min}$ of on-source integration time, taken while the $1.5 \mathrm{~mm}$ system temperature varied from 400 to $450 \mathrm{~K}$. The deterioration of the weather conditions prevented the acquisition of more data. The individual scans were examined, stacked, and binned to $47 \mathrm{~km} \mathrm{~s}^{-1}$ channels within the CLASS environment ${ }^{1}$.

We followed up 4C12.50 with the PACS (Poglitsch et al. 2010) far-infrared instrument onboard Herschel (Pilbratt et al. 2010) to look for emission from $\mathrm{CO}$ molecules that are excited to states of high rotational number. High spectral sampling $\mathrm{CO}(16-15)$ observations were carried out for our GT2_lspinogl_6 program in range-spectroscopy mode using chop-nod cycles. To populate the $\mathrm{CO}$ spectral line energy distribution (SLED), we combined our data with all PACS

http://www.iram.fr/IRAMFR/GILDAS 
range or line spectroscopy data in the Herschel archive that cover the observed-frame wavelengths of $\mathrm{CO}$ lines in $4 \mathrm{C} 12.50$. For this reason, we examined the blue array data and their parallel red array data in each astronomical observation request (AOR). We found that, in total, information is available for four transitions, $\mathrm{CO}(33-32), \mathrm{CO}(22-21), \mathrm{CO}(18-17)$, and $\mathrm{CO}(16-15)$, with integration times of $1.2,4.0,1.4$, and $2.1 \mathrm{~h}$, respectively. For $\mathrm{CO}(22-21)$, this is the total time of the combined OT1_dfarrah_1 and OT1_sveilleu_3 program data. We reduced the data within the Herschel Interactive Processing Environment (HIPE; version 8.3, calibration tree version 32; Ott et al. 2010), by running the default PACS spectrometer pipeline for chopped-line and short-range scans with a wavelength grid oversampling parameter ${ }^{2}$ of 2 . We flagged as outliers all pixel read-outs in emission-line-free wavelengths whose flux exceeded $5 \sigma$. Because $4 \mathrm{C} 12.50$ is a point source for PACS, we obtained its final spectrum at each wavelength by extracting the spectrum of the central $9.4^{\prime \prime} \times 9.4^{\prime \prime}$ spaxel of each data cube and by multiplying it by the point-source-correction factor in order to recover its full flux. We followed an identical procedure to reduce all other archival PACS observations of 4C12.50 in order to obtain information on its far-infrared dust continuum emission. From this process, we excluded AORS in spectral ranges that suffer from flux leakage from/to other wavelengths, such as $<54 \mu \mathrm{m}, 70-74 \mu \mathrm{m}$, and 95-105 $\mu \mathrm{m}$.

\section{Results}

\section{1. $C O(1-0)$ data: gas mass and distribution}

The median channel with a $\mathrm{CO}(1-0)$ detection in the $\mathrm{PdB}$ data cube is at $102.756 \mathrm{GHz}$, pointing to a redshift $z$ of 0.12179 . When adopting this redshift and collapsing the continuumsubtracted cube over the $-500 \mathrm{~km} \mathrm{~s}^{-1}$ to $500 \mathrm{~km} \mathrm{~s}^{-1}$ velocity range, we find that the $\mathrm{CO}(1-0)$ emission is offset from the $3 \mathrm{~mm}$ continuum peak and marginally extended (Fig. 1). When examining the data cube within the $-500<V<500 \mathrm{~km} \mathrm{~s}^{-1}$ range, we find that the $\mathrm{CO}(1-0)$ emission progresses from the east to the west as the velocity increases from negative to positive values (Figs. 2, 3). It peaks at two locations, $\sim 1^{\prime \prime}$ (or $\sim 2.2 \mathrm{kpc}$ ) apart. This emission arises from gas that could be associated with the two main merging nuclei of 4C12.50 (Dasyra et al. 2006a), with an off-center disk forming due to the merger, or with a combination of the above.

A distinct, off-center component is also detected in the central velocity bin of Fig. 3, at $\sim 10 \mathrm{kpc}$ southwest of the main nucleus. An inspection of the data cube at a higher spectral resolution (Fig. 4) reveals that this component has a tidal structure, approaching the main component at systemic velocity. Its distance from the center progressively increases with increasing velocity, until it reaches $12 \mathrm{kpc}$ at $\sim 30 \mathrm{~km} \mathrm{~s}^{-1}$. The structure has a faint counterpart in the optical (Fig. 5), and it is by far the most gas-rich (and thus dust-rich) of all tidal tails in this system.

The spectra extracted from the data cube are shown in Figs. 6 and 7 for the main component, and Fig. 8 for the tidal tail. Figure 6 reveals an asymmetry in the $\mathrm{CO}(1-0)$ emission profile along the east-west axis at sub-beam scales that is likely due to the merger. The profile can also be compatible with absorption of $\mathrm{CO}$ and continuum emission by molecules at systemic

\footnotetext{
2 The differences between the spectra obtained by running the default pipeline and the spectra obtained by running the routines for an alternative flux calibration based on the telescope background are within the given error bars.
}

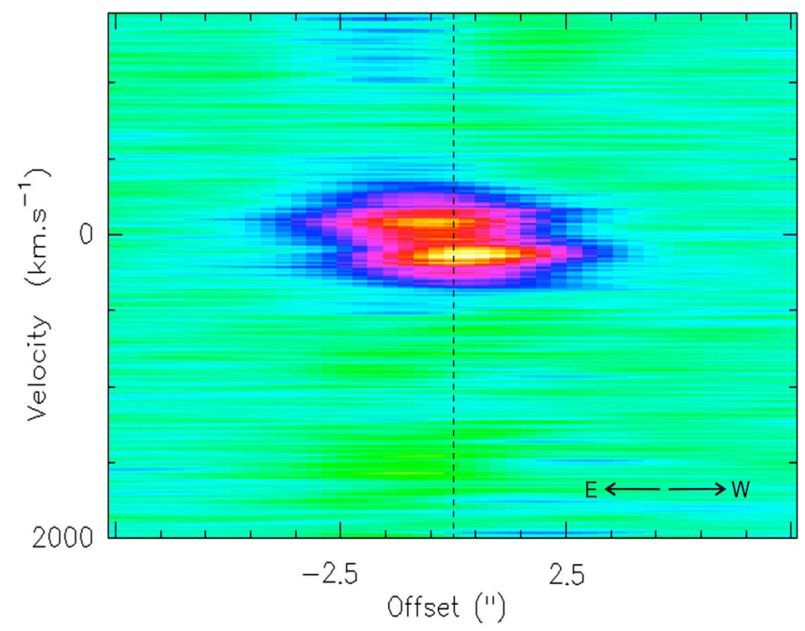

Fig. 2. Position-velocity diagram of the continuum-free $\mathrm{CO}(1-0)$ emission along the east-west axis, displayed for a velocity resolution of $6 \mathrm{~km} \mathrm{~s}^{-1}$. The $x$-axis offset is computed from the radio core location, which is marked with a dashed line. The offset increases (/decreases) toward the west (/east).

velocity, such as in Centaurus A (Eckart et al. 1990; Wiklind \& Combes 1997).

To find the mass of the $\mathrm{H}_{2}$ gas that is associated with the $\mathrm{CO}$ emission shown in Figs. 7 and 8, we computed the product $3.25 \times 10^{7} \alpha S_{\mathrm{CO}(1-0)} \Delta V v_{\mathrm{obs}}^{-2} D_{L}^{2} /(1+z)^{3} M_{\odot}$, where $\alpha$ is the $\mathrm{CO}$ intensity to $\mathrm{H}_{2}$ mass conversion factor, $S_{\mathrm{CO}(1-0)} \Delta V$ is the integrated line flux in $\mathrm{Jy} \mathrm{km} \mathrm{s}^{-1}, v_{\mathrm{obs}}$ is the observed $\mathrm{CO}(1-0)$ frequency in $\mathrm{GHz}$, and $D_{L}$ is the source luminosity distance in Mpc (Solomon et al. 1997). We adopted an $\alpha$ value of $0.8 M_{\odot} /\left(\mathrm{K} \mathrm{km} \mathrm{s}^{-1} \mathrm{pc}^{2}\right)$, to be consistent with the literature assuming a lower conversion factor for ultraluminous infrared galaxies (ULIRGs) than for the Milky Way (Downes \& Solomon 1998).

For the main emission component (Figs. 1, 7), we measured a flux of $18.2 \pm 1.5 \mathrm{Jy} \mathrm{km} \mathrm{s}^{-1}$. From this flux we calculated a cold $\mathrm{H}_{2}$ gas mass reservoir of $1.0( \pm 0.1) \times 10^{10} M_{\odot}$. Likewise, the flux in the tidal tail is $0.61 \pm 0.04 \mathrm{Jy} \mathrm{km} \mathrm{s}^{-1}$ (integrated down to the $1 \sigma$ level, as shown in Fig. 5). This corresponds to an $\mathrm{H}_{2}$ mass of $3.3( \pm 0.3) \times 10^{8} M_{\odot}$. We thus find that a mass comparable to $1 / 30^{\text {th }}$ of the main reservoir could be infalling thanks to the tidal tail (see Sect. 4). Because the total CO(1-0) flux in the PdB data agrees within the error with that previously measured in IRAM $30 \mathrm{~m}$ telescope data (20 $\pm 6 \mathrm{Jy} \mathrm{km} \mathrm{s}^{-1}$; Dasyra \& Combes 2011), we deduce that the interferometer did not miss a significant part of the extended emission.

A spatially-unresolved absorption line at $-720 \mathrm{~km} \mathrm{~s}^{-1}$ at the radio core and less than a handful of emission lines at $V<$ $-500 \mathrm{~km} \mathrm{~s}^{-1}$ or $V>500 \mathrm{~km} \mathrm{~s}^{-1}$ were seen in the PdB data at $\sim 3-5$ root mean square noise $(\sigma)$ levels. Their widths never exceeded $\sim 80 \mathrm{~km} \mathrm{~s}^{-1}$. These lines could be real (owing to a small collection of high-velocity clouds in the outflow) or artifacts (due to the varying position and intensity of the $\mathrm{mm}$ continuum, which leads to an inadequate removal of the point spread function). A reliable detection of the outflow in $\mathrm{CO}(1-0)$ remains to be achieved with higher dynamic range observations. In the rest of this work, we adopt an upper limit of $1.3 \times 10^{8} M_{\odot}$ for the mass of the cold molecular gas in the outflow. We computed this limit by integrating $3 \sigma$ over the velocity range that the molecular outflow spans in other wavelengths $\left(-1300 \mathrm{~km} \mathrm{~s}^{-1}<\right.$ $V<-300 \mathrm{~km} \mathrm{~s}^{-1}$; Dasyra \& Combes 2011, 2012). We further assumed that the outflow is spatially unresolved, constrained 


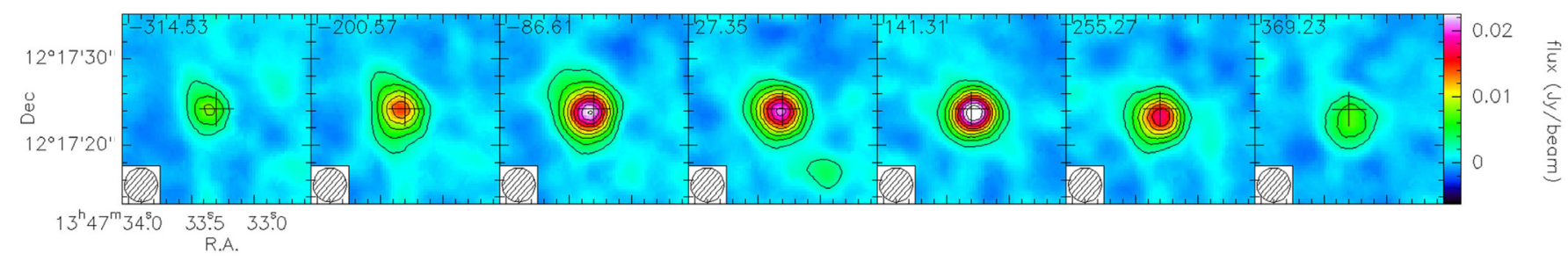

Fig. 3. Continuum-free $\mathrm{CO}(1-0)$ line intensity map of $4 \mathrm{C} 12.50$, displayed for $\sim 114 \mathrm{~km} \mathrm{~s}^{-1}$ bins (in the range $-315 \mathrm{~km} \mathrm{~s}^{-1}$ to $369 \mathrm{~km} \mathrm{~s}^{-1}$ ). The number in the upperleft corner of each frame corresponds to the bin mean velocity. Contours are plotted at integer multiples of the $5 \sigma$ level for these images, i.e., in steps of $2.4 \mathrm{mJy} / \mathrm{beam}$. A shift toward the west of the line peak position from negative to positive velocities, as well as a distinct kinematic component near rest-frame velocity are observed.

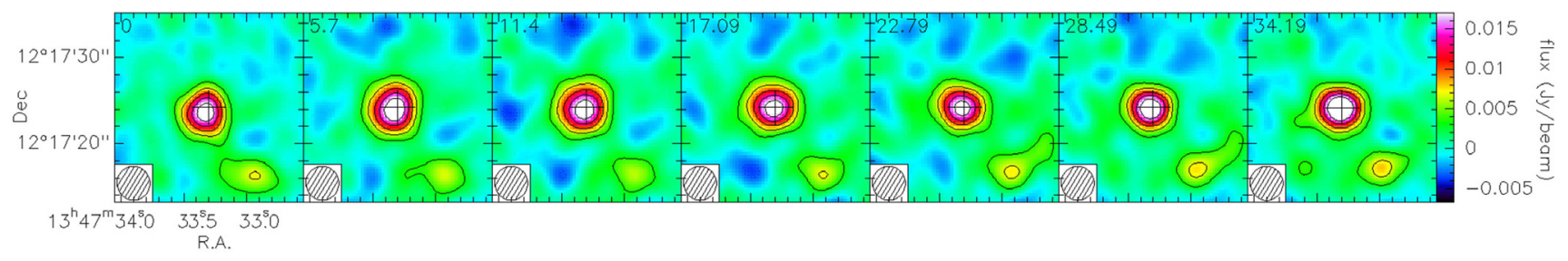

Fig. 4. Same as in Fig. 3, but for bins of $17 \mathrm{~km} \mathrm{~s}^{-1}$ (in the range 0 to $34 \mathrm{~km} \mathrm{~s}^{-1}$ ). Contours are plotted at integer multiples of the $3.5 \sigma$ level for these images, i.e., in steps of $3.3 \mathrm{mJy} / \mathrm{beam}$.

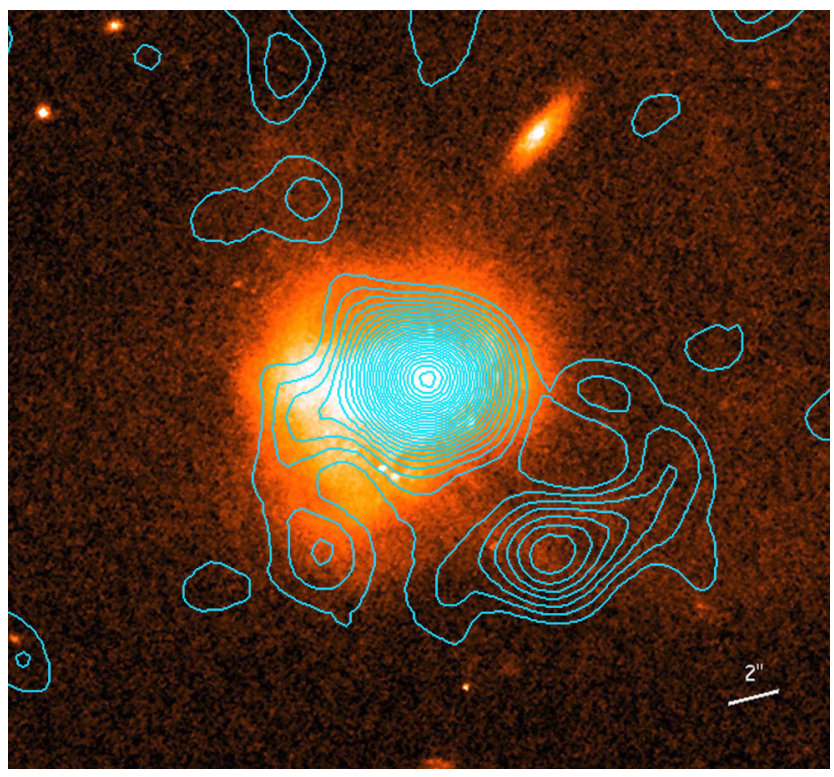

Fig. 5. Contours of the $\mathrm{CO}(1-0)$ emission of $4 \mathrm{C} 12.50$, integrated from $25 \mathrm{~km} \mathrm{~s}^{-1}$ to $35 \mathrm{~km} \mathrm{~s}^{-1}$ and plotted over an $7300 \AA$ Hubble Space Telescope ACS image (Batcheldor et al. 2007). The contours start at the $1 \sigma$ level of the collapsed $\mathrm{CO}(1-0)$ image, $0.014 \mathrm{Jy} / \mathrm{beam}$. They are spaced by an equal amount.

within our four-kiloparsec-radius beam. Indeed, no evidence of an extended (ionized gas) outflow was found in multi-slit optical spectra of 4C12.50 (Holt et al. 2008, 2011). The neutral atomic gas component of the outflow observed in the radio is found $\sim 150 \mathrm{pc}$ away from the nucleus, at the tip of the southern radio hot spot (Morganti et al. 2013b).

\subsection{Higher rotational number $\mathrm{CO}$ lines}

The IRAM $30 \mathrm{~m}$ telescope $\mathrm{CO}(2-1)$ data (Fig. 9) have a beam of $\sim 12^{\prime \prime}$, which comprises all regions seen in emission in the
PdB data. The measured CO(2-1) flux ${ }^{3}$ was $35( \pm) 6 \mathrm{Jy} \mathrm{km} \mathrm{s}^{-1}$, which together with a previously measured $\mathrm{CO}(3-2)$ flux of $50 \pm 8 \mathrm{Jy} \mathrm{km} \mathrm{s}^{-1}$ (Dasyra \& Combes 2012), indicates a subthermal excitation of the $\mathrm{CO}$ molecules. Their excitation up to $J=3$ is identical with that of the gas in the Milky Way, toward the Galactic center, and higher than in the outflow of M82 (Fig. 11; Weiss et al. 2001, 2005).

High- $J$ CO emission was not detected with PACS, with a probable exception for the 22-21 transition (Fig. 10): weak $(3.5 \sigma)$ signal was seen at restframe $118.58 \mu \mathrm{m}$, which was also seen by Spoon et al. (2013) and Veilleux et al. (2013) in their independent reductions of different sub-sets of the data. For the sake of completeness, we note that a spectrum of $4 \mathrm{C} 12.50$ showing no detection of CO lines was also acquired (OT1_pogle01_1) with SPIRE (Griffin et al. 2010). The limits used in this work (Table 1) are based on the wavelength-dependent instrumental sensitivity for the integration time of the SPIRE observations.

By means of a qualitative comparison of the SLED (Fig. 11), we find that the high- $J$ states of $\mathrm{CO}$ molecules are considerably less populated in 4C12.50 than those in local prototype AGN in which shock fronts and X-ray-photodissociation regions are likely to contribute to the gas excitation (e.g., NGC 1068 and NGC 6240; Krips et al. 2011; Hailey-Dunsheath et al. 2012; Spinoglio et al. 2012; Meijerink et al. 2013).

\subsection{CO spectral line energy distribution fitting: cold vs. warm gas reservoir}

To derive fundamental properties of the gas that is probed by our CO observations, we modeled the CO SLED with the radiative transfer code RADEX, assuming for simplicity that the flux

3 The width of the $\mathrm{CO}(2-1)$ line in the $30 \mathrm{~m}$ telescope data, $390 \pm$ $68 \mathrm{~km} \mathrm{~s}^{-1}$, is lower and more uncertain than that of the $\mathrm{CO}(1-0)$ line in the PdB data. The uncertainty is due to the sinusoidal baseline in the single-dish data due to standing waves related to the bright continuum of $4 \mathrm{C} 12.50$. It does not necessarily translate into a flux loss. For comparison, see the different line shape but identical line flux of $\mathrm{CO}(1-0)$ obtained with the PdB array and the $30 \mathrm{~m}$ telescope (Dasyra \& Combes 2012). 


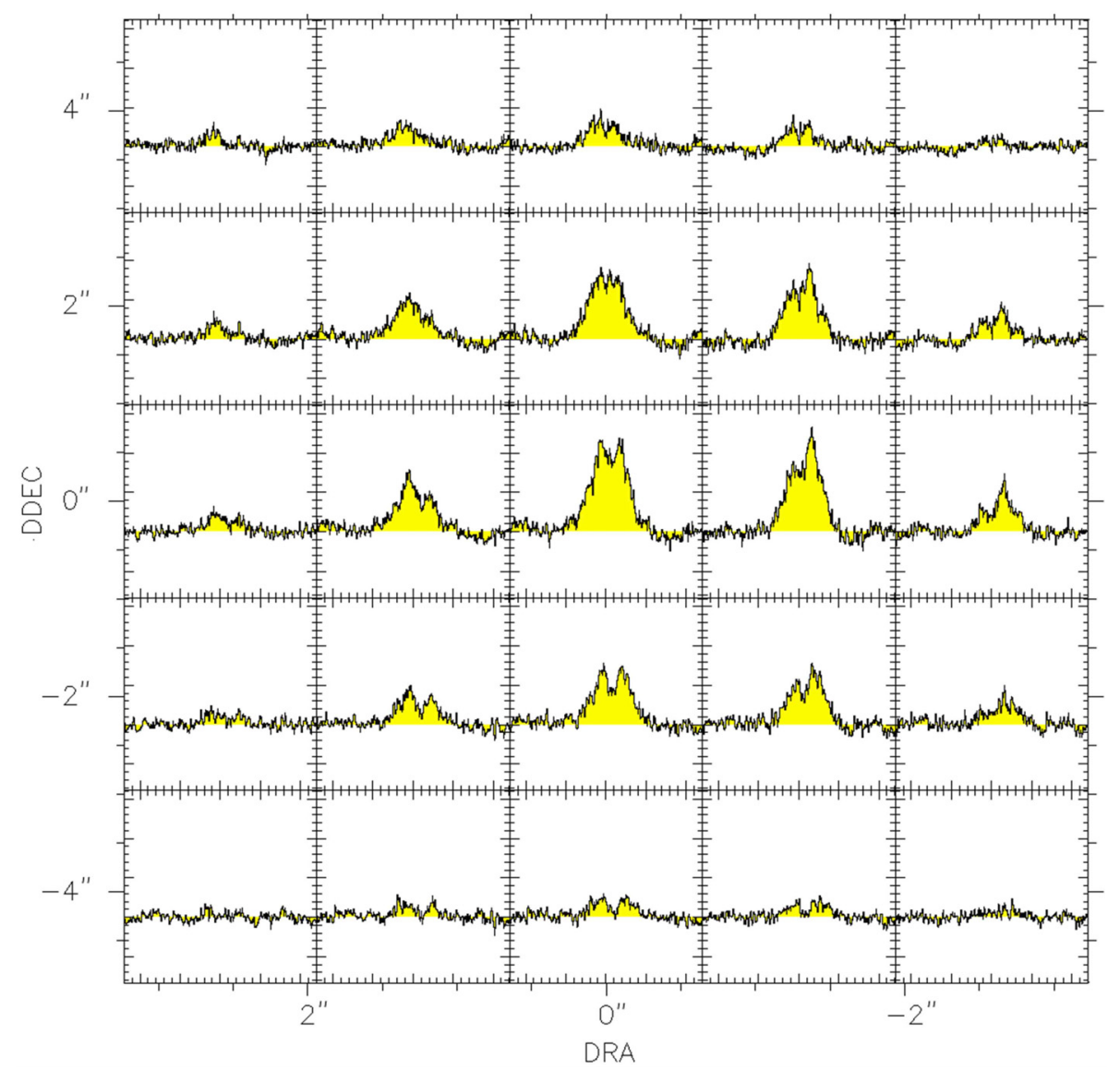

Fig. 6. $\mathrm{CO}(1-0)$ line profile changes within few arcseconds (i.e., at sub-beam scales) from the position of the radio core in the $\mathrm{PdB}$ data. All spectra are plotted in the $-1200 \mathrm{~km} \mathrm{~s}^{-1}$ to $1200 \mathrm{~km} \mathrm{~s}^{-1}$ velocity range.

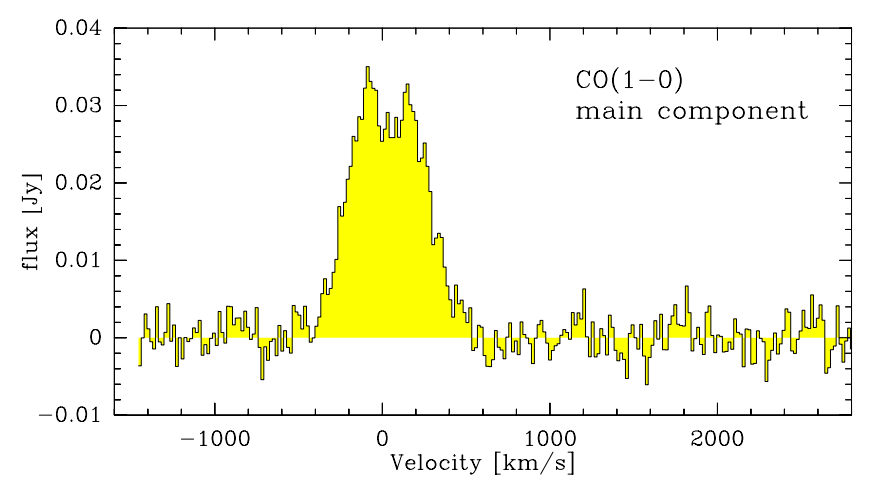

Fig. 7. Line profile of the main $\mathrm{CO}(1-0)$ emission component in the $\mathrm{PdB}$ data. The flux is integrated down to the $1 \sigma$ level of the integrated $\mathrm{CO}(1-0)$ emission (Fig. 1; typically 7-9" from the radio core), excluding the emission from the tidal structure, which is shown in Fig. 8.

that we detected over a line width of $\sim 520 \mathrm{~km} \mathrm{~s}^{-1}$ originates in a collection of clouds with same average properties and intrinsic line widths of $1 \mathrm{~km} \mathrm{~s}^{-1}$ (van der Tak et al. 2007). Further assuming that the cosmic microwave background temperature is $3.06 \mathrm{~K}$ and that the $\mathrm{CO}$ is excited by collisions with hydrogen and helium, we ran a grid of $\mathrm{CO}$ column density $N_{\mathrm{CO}}$, CO kinetic temperature $T_{\text {kin }}$, and $\mathrm{H}_{2}$ volume density $n_{\mathrm{H} 2}$ models to find those that best fit our data.

We considered intrinsic column densities in the range $5 \times$ $10^{14}-5 \times 10^{17} \mathrm{~cm}^{-2}$, corresponding to the beam-averaged CO column density in the chosen velocity step, divided by a filling factor of the beam by the clouds in the range $1-0.001$. To compute this beam-averaged column density from the $\mathrm{CO}(1-0)$

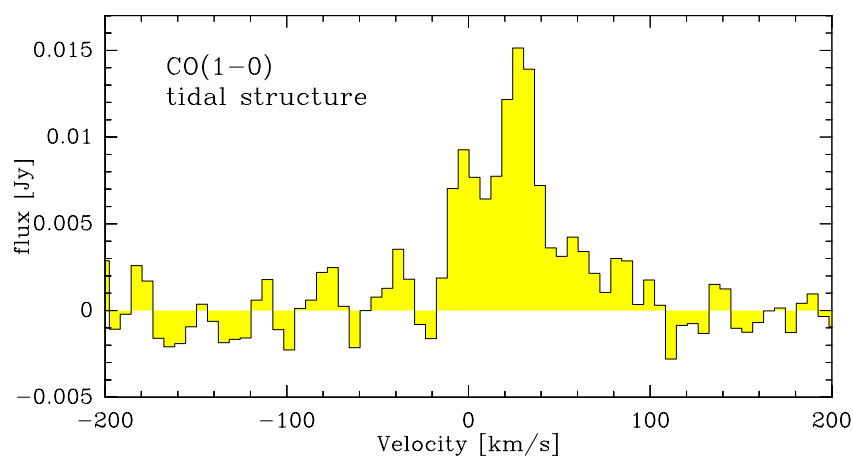

Fig. 8. PdB $\mathrm{CO}(1-0)$ spectrum of the tidal structure southwest of the merging nuclei of $4 \mathrm{C} 12.50$, integrated down to the $1 \sigma$ flux level of Fig. 5.

observations, we used an $\mathrm{H}_{2}$ gas mass of $1.0 \times 10^{10} M_{\odot}$, a $\mathrm{CO}$ abundance that is $10^{4}$ times lower than that of the $\mathrm{H}_{2}$, and we equally divided the mass between 520 velocity bins. Having three unknown parameters and three line luminosity measurements, we obtained an exact solution for the model that best describes the molecular emission from the $J=1, J=2$, and $J=3$ upper states. It corresponds to a filling factor of 0.025 , a $\mathrm{CO}$ kinetic temperature of $25 \mathrm{~K}$, and an $\mathrm{H}_{2}$ volume density of $1.5 \times 10^{3} \mathrm{~cm}^{-3}$. In this solution, the $\mathrm{CO}(1-0)$-emitting gas is out of local thermodynamic equilibrium (LTE): the $\mathrm{CO}(1-0)$ beam-corrected brightness temperature is $11 \mathrm{~K}$, i.e., $\sim$ two times lower than the $\mathrm{H}_{2}$ kinetic temperature. The data of the $\mathrm{CO}(1-0)$ and $\mathrm{CO}(2-1)$ lines alone are compatible with a temperature of $7 \mathrm{~K}$, for the same volume density but for a higher filling factor $(0.092)$. The data of the $\mathrm{CO}(1-0)$ and $\mathrm{CO}(3-2)$ lines alone 


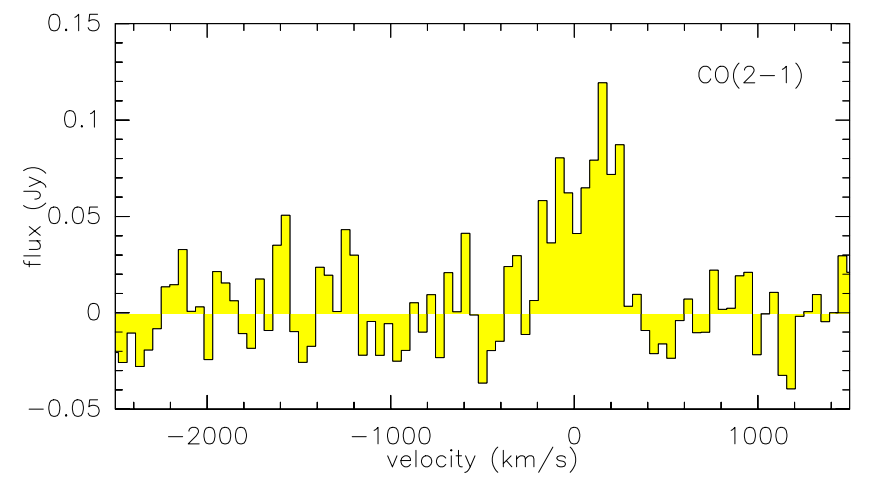

Fig. 9. IRAM $30 \mathrm{~m}$ telescope data of $\mathrm{CO}(2-1)$, comprising all regions where $\mathrm{CO}(1-0)$ was detected in the $\mathrm{PdB}$ data (i.e., the main component and the tidal structure).

Table 1. CO line observations.

\begin{tabular}{lccc}
\hline \hline Line & $\begin{array}{c}\lambda_{\text {restframe }} \\
(\mu \mathrm{m})\end{array}$ & $\begin{array}{c}\text { Flux } \\
\left(10^{-18} \mathrm{~W} \mathrm{~m}^{-2}\right)\end{array}$ & $\begin{array}{c}F W H M \\
\left(\mathrm{~km} \mathrm{~s}^{-1}\right)\end{array}$ \\
\hline $\mathrm{CO}(1-0)$ & 2600.7 & $0.064 \pm 0.005$ & $517 \pm 14$ \\
$\mathrm{CO}(2-1)$ & 1300.4 & $0.24 \pm 0.04$ & $390 \pm 68$ \\
$\mathrm{CO}(3-2)$ & 866.96 & $0.51 \pm 0.08^{1}$ & $510 \pm 65^{1}$ \\
$\mathrm{CO}(5-4)$ & 520.23 & $<8.0^{2}$ & $\ldots$ \\
$\mathrm{CO}(6-5)$ & 433.55 & $<4.7^{2}$ & $\ldots$ \\
$\mathrm{CO}(7-6)$ & 371.65 & $<3.5^{2}$ & $\ldots$ \\
$\mathrm{CO}(8-7)$ & 325.22 & $<4.4^{2}$ & $\ldots$ \\
$\mathrm{CO}(9-8)$ & 289.12 & $<5.8^{2}$ & $\ldots$ \\
$\mathrm{CO}(10-9)$ & 260.24 & $<6.0^{2}$ & $\ldots$ \\
$\mathrm{CO}(11-10)$ & 236.61 & $<6.0^{2}$ & $\ldots$ \\
$\mathrm{CO}(12-11)$ & 216.93 & $<6.0^{2}$ & $\ldots$ \\
$\mathrm{CO}(13-12)$ & 200.27 & $<6.0^{2}$ & $\ldots$ \\
$\mathrm{CO}(16-15)$ & 162.81 & $<2.5$ & $\ldots$ \\
$\mathrm{CO}(18-17)$ & 144.78 & $<1.8$ & $\ldots$ \\
$\mathrm{CO}(22-21)$ & 118.58 & $0.97 \pm 0.27$ & $\ldots$ \\
$\mathrm{CO}(33-32)$ & 79.36 & $<5.0$ & $\ldots$ \\
\hline
\end{tabular}

Notes. ${ }^{(1)}$ Value taken from Dasyra \& Combes (2012). ${ }^{(2)}$ Estimated from SPIRE's sensitivity for $2.8 \mathrm{~h}$ on source.

are compatible with a temperature as high as $32 \mathrm{~K}$, again for the same volume density but for a lower filling factor (0.017).

To estimate the maximum warm gas mass that is probed by the $\mathrm{CO}$, we calculated the maximum emission by molecules at high rotational states that is compatible with the observed SLED after fixing $T_{\text {kin }}$ to $400 \mathrm{~K}$. This kinetic temperature is marginally higher than the excitation temperature of the $\mathrm{H}_{2}$ gas seen with Spitzer $(374 \pm 12 \mathrm{~K}$; Dasyra \& Combes 2011). For this computation, our free parameters were the $\mathrm{H}_{2}$ volume density and the $\mathrm{CO}$ column density. Our column density grids were expanded to lower values than before. A model fitting the $\mathrm{CO}(18-17)$ and $\mathrm{CO}(22-21)$ data has an $\mathrm{H}_{2}$ volume density of $2 \times 10^{6} \mathrm{~cm}^{-3}$, and a CO column density of $5 \times 10^{13} \mathrm{~cm}^{-2}$ (Fig. 11). The mass of the warm $\mathrm{H}_{2}$ as probed by the $\mathrm{CO}$ at $400 \mathrm{~K}$ is then equal to $2.5 \times 10^{7} M_{\odot}$, or 0.0025 times the mass of the cold $\mathrm{H}_{2}$ probed by the $\mathrm{CO}$ at $25 \mathrm{~K}$, under the assumption that the emitting areas of the warm and the cold gas are identical. The $400 \mathrm{~K}$ gas mass would decrease if the $\mathrm{CO}(22-$ 21) line luminosity were to be treated as an upper limit. If the warm gas were to be more tenuous, with an $\mathrm{H}_{2}$ volume density of $1 \times 10^{5} \mathrm{~cm}^{-3}$, then the $\mathrm{CO}$ column density would have to be
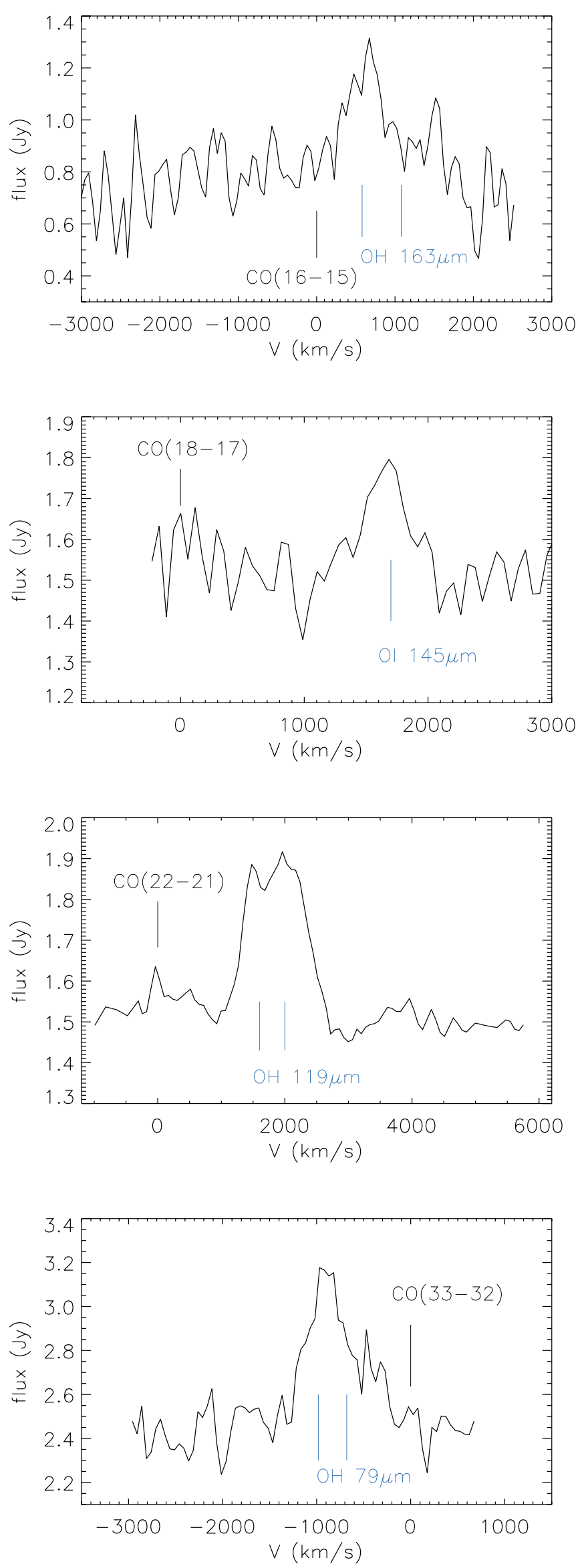

Fig. 10. PACS observations of $4 \mathrm{C} 12.50$ that cover the wavelengths of high- $J$ CO spectral lines. 
K. M. Dasyra et al.: Heating of the molecular gas in the massive outflow of $4 \mathrm{C} 12.50$

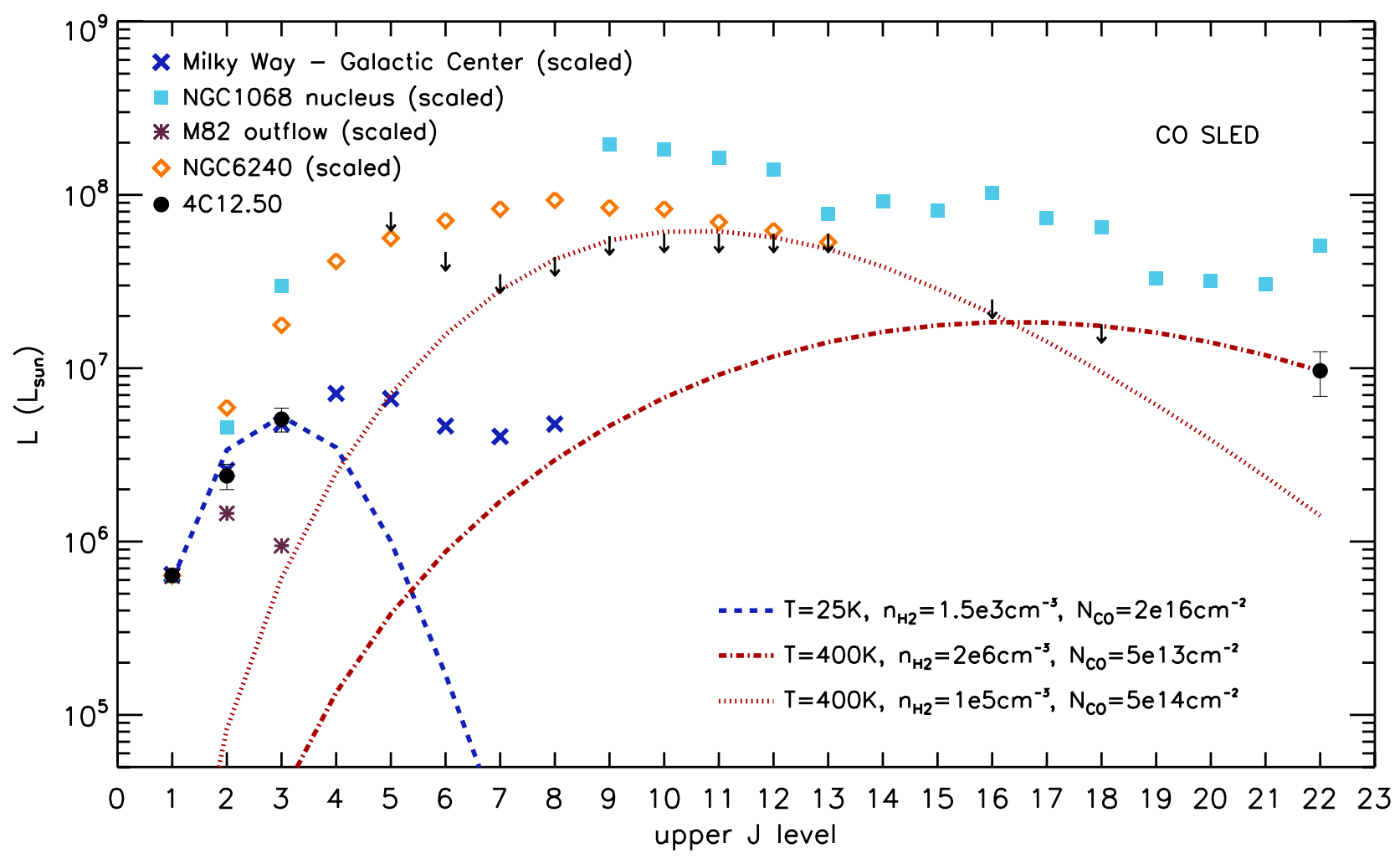

Fig. 11. CO SLED of $4 \mathrm{C} 12.50$ shown for transitions up to $J=22-21$. The $\mathrm{CO}(22-21)$ line is seen at $3.5 \sigma$ levels. Based on our RADEX modeling, the SLED of the three lowest transitions is best fit by gas with a kinetic temperature of $25 \mathrm{~K}$ (dashed line). Examples of RADEX models that are consistent with the Herschel data are also shown for gas with a kinetic temperature of $400 \mathrm{~K}$, for a couple of column and volume density combinations (dashed-dotted and dotted lines). The SLEDs of other prototype sources are overplotted for comparison, normalized to $L_{\mathrm{CO}(1-0)}(4 \mathrm{C} 12.50) / \mathrm{L}_{\mathrm{CO}(1-0)}$.

below $5 \times 10^{14} \mathrm{~cm}^{-2}$ for the $\mathrm{CO}(7-6)$ to $\mathrm{CO}(13-12)$ luminosity limits to be respected. In that case, the upper limit on the mass of the warm gas would be $2.5 \times 10^{8} M_{\odot}$. In the likely presence of intermediate $T_{\text {kin }}$ components, the mass estimate will again decrease.

To compare the gas and dust properties in the ambient medium, we produced the dust spectral energy distribution (SED) of 4C12.50 using our Herschel and IRAM continuum flux measurements (from this work, Table 2, Dasyra \& Combes 2012) together with data from the literature (Moshir et al. 1990; Steppe et al. 1995; Clements et al. 2010; Ostorero et al. 2010; Trippe et al. 2010; Guillard et al. 2012). The large scatter in the radio data is due to the time variability of the continuum. We fitted the SED by minimizing the $\chi^{2}$ between the observations and a model consisting of multiple modified black body curves and a power law. We parameterized the flux emitted by each modified black body as $\left(M_{\text {dust }} K_{\text {abs }}\left[\lambda_{0}\right] / D_{L}^{2}\right)\left(\lambda_{0} / \lambda\right)^{\beta}\left(2 h v^{3} / c^{2}\right) /\left(\mathrm{e}^{h v / k T}-1\right)$, where $M_{\text {dust }}$ is the dust mass at temperature $T, \kappa_{\mathrm{abs}}\left[\lambda_{0}\right]$ is the dust absorption coefficient at the reference wavelength $\lambda_{0}, h$ is the Planck constant, and $k$ is the Boltzmann constant. We opted for a reference wavelength accessible to PACS, $150 \mu \mathrm{m}$, and parameter values of $\beta=2.0$ and $\kappa_{\mathrm{abs}}[150 \mu \mathrm{m}]=11.6 \mathrm{~cm}^{2} \mathrm{gr}^{-1}$. These parameter values reproduce the absorption coefficient of the Draine (2003) $R_{V}=3.1$, Milky Way dust model with an accuracy of $10 \%$ in the 70 to $1000 \mu \mathrm{m}$ range. We limited the temperature of the coldest dust SED component in the range 5-35 K. We also fixed the temperature of another component at $400 \mathrm{~K}$, i.e., at the $T_{\text {kin }}$ of the warm CO SLED component. A $\chi^{2}$ minimization using the IDL MPFIT code indicated that three more components are
Table 2. Continuum fluxes in the infrared and $\mathrm{mm}$ range.

\begin{tabular}{lc}
\hline \hline $\begin{array}{l}\lambda_{\text {restframe }} \\
(\mu \mathrm{m})\end{array}$ & $\begin{array}{c}\text { Flux } \\
(\mathrm{Jy})\end{array}$ \\
\hline 52.0 & $2.21 \pm 0.10$ \\
57.5 & $2.39 \pm 0.15$ \\
78.7 & $2.46 \pm 0.11$ \\
81.0 & $2.30 \pm 0.18$ \\
121.0 & $1.51 \pm 0.06$ \\
126.0 & $1.53 \pm 0.10$ \\
146.0 & $1.48 \pm 0.12$ \\
155.0 & $1.36 \pm 0.17$ \\
158.5 & $1.15 \pm 0.10$ \\
162.5 & $0.85 \pm 0.13$ \\
172.0 & $1.00 \pm 0.19$ \\
870 & $0.15 \pm 0.02$ \\
1305 & $0.30 \pm 0.07$ \\
2610 & $0.51 \pm 0.06$ \\
\hline
\end{tabular}

needed to reproduce the observed SED (Fig. 12). From the SED modeling, we found that the infrared luminosity of $4 \mathrm{C} 12.50$ is $2.4( \pm 0.1) \times 10^{12} L_{\odot}$, that the component that best fits the peak of the SED is at $T=40 \pm 3 \mathrm{~K}$, and that the component with the lowest temperature is at $19 \pm 5 \mathrm{~K}$.

The temperatures of the coldest dust SED components thus bracket the kinetic temperature of the cold CO SLED component $(25 \pm 8 \mathrm{~K})$. The SLED could also be reproduced up to $\mathrm{CO}(3-2)$ by a combination of two gas components with temperatures lower 


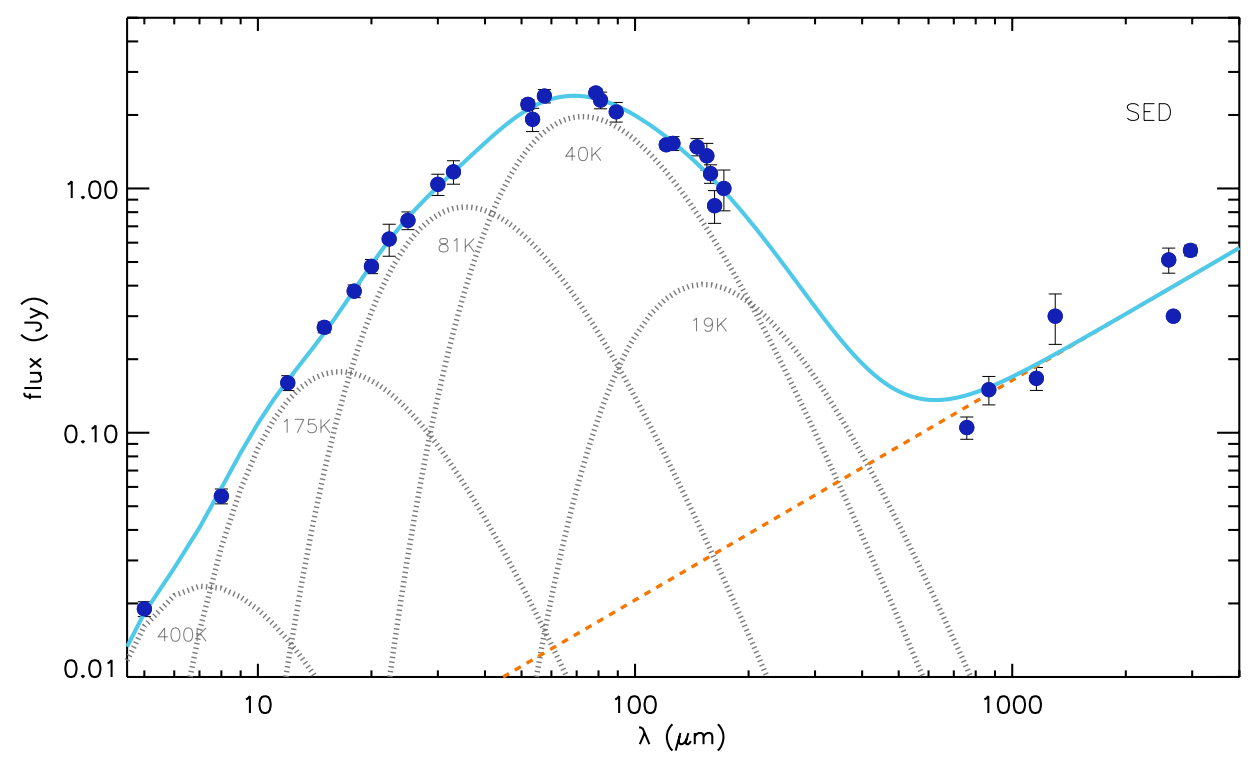

Fig. 12. Dust SED of $4 \mathrm{C} 12.50$ including continuum measurements from the new IRAM and Herschel data. The modified black body curves (with temperatures between $19 \mathrm{~K}$ and $400 \mathrm{~K}$ ) that best fit the IR/sub-mm data are plotted with dotted lines. The dashed line is a synchrotronrelated power law with an exponent of 0.9 . The solid line corresponds to the sum of the flux of all components. than or equal to those of the dust (e.g., $7 \mathrm{~K}$ and $40 \mathrm{~K}$ ). Collisions with the ambient ISM could thus suffice to explain the excitation of the bulk of the $\mathrm{CO}$ molecules. A jet-induced shock does not have to be invoked instead.

\section{Discussion}

\subsection{Heating of the accelerated molecular gas with respect to the ambient medium}

The results presented in Sect. 3 raise the question of how the excitation properties of the accelerated molecular gas compare with those of the dynamically relaxed gas. From the Spitzer data, we know that the outflow has a warm component, carrying $5.2 \times 10^{7} M_{\odot}$ of $\mathrm{H}_{2}$ molecules at $\sim 400 \mathrm{~K}$ (Dasyra \& Combes 2011). From a $\mathrm{CO}(2-3)$ absorption line that is due to clouds obscuring the millimeter continuum and moving at $-950( \pm 90) \mathrm{km} \mathrm{s}^{-1}$, we know that colder molecules do exist in the outflow (Dasyra \& Combes 2012). Only a lower limit could be placed on their mass from the $\mathrm{CO}(2-3)$ data, because the absorption only probes the few lines of sight toward the millimeter continuum. The millimeter continuum is less extended than the radio continuum due to the steep power law that describes the emission from the jet lobes (Krichbaum et al. 1998, 2001, 2008), and the radio continuum is in its turn constrained to around ten hot spots with a radius of a few parsec each (Morganti et al. $2013 \mathrm{~b}$ ). The bulk of the cold entrained CO is thus likely to be found in lines of sight without a millimeter background, and it should be detected in emission. Its non-detection in our $\mathrm{CO}(1-0)$ $\mathrm{PdB}$ data now enables us to place an upper limit of $1.3 \times 10^{8} M_{\odot}$ on the mass of cold entrained $\mathrm{H}_{2}$ gas.

Putting all numbers together, we find that the mass ratio of warm $(400 \mathrm{~K})$ to cold $(25 \mathrm{~K}) \mathrm{H}_{2}$ gas in the outflow is $>0.40$. For the ambient medium, containing $1.0 \times 10^{10} M_{\odot}$ of $\mathrm{H}_{2}$ molecules at $25 \mathrm{~K}$ and $1.4 \times 10^{8} M_{\odot}$ of $\mathrm{H}_{2}$ molecules at $\sim 400 \mathrm{~K}$ (Sect. 3; Dasyra \& Combes 2011), the same ratio is only 0.014. A major result of this work is thus that the mass ratio of warm-tocold $\mathrm{H}_{2}$ gas is at least 29 times higher in the outflow than in the ambient medium.

Taking into account that a non-negligible fraction of the midinfrared $\mathrm{H}_{2}$ emission could be due to gas that was accelerated by the front shock but that is not accounted for in the outflow due to its low velocity, we conclude that the discrepancy in the warmto-cold mass ratio could increase. Contrarily, adding the mass of the warm ambient $\mathrm{H}_{2}$ probed by high- $J \mathrm{CO}$ lines to the mass of the warm ambient $\mathrm{H}_{2}$ measured by Spitzer could decrease this discrepancy. Still, it would not alter our conclusion. Adding $2.5 \times 10^{7} M_{\odot}$ of gas distributed in a dense $400 \mathrm{~K}$ component (in agreement with the high- $J \mathrm{CO}$ emission in Fig. 11) would lead to a warm-to-cold $\mathrm{H}_{2}$ mass ratio that is $\geq 24$ times higher in the outflow than in the ambient medium. Even adding the maximum amount of $400 \mathrm{~K}$ gas that is compatible with the CO SLED, $2.5 \times 10^{8} M_{\odot}$, would lead to a ratio that is $\geq 10$ times higher in the outflow than in the ambient medium. Likewise, our conclusion is robust against the use of another $\alpha$ factor, or the use of different $\alpha$ factors for the entrained and ambient gas (see, e.g., Papadopoulos et al. 2012). The latter choice can be justified because the $\mathrm{CO}$ intensity to $\mathrm{H}_{2}$ mass conversion scales with $T_{\text {kin }}{ }^{-1}\left(n_{\mathrm{H} 2}\right)^{1 / 2}$ for virialized clouds (Maloney et al. 1988; Weiss et al. 2001). Figure 13, showing the dependence of the warm-tocold $\mathrm{H}_{2}$ mass ratio on the $\alpha$ factor, indicates that there is no combination of acceptable $\alpha$ and warm gas mass values that could make the ratio in the ambient medium agree with the one in the outflow. Instead, the ratio in the outflow is at least 3-30 times higher than in the ambient medium in the entire parameter space.

Our results constitute direct evidence that the accelerated molecular gas can be heated ${ }^{4}$ to the point where star formation is inefficient. The accelerated and heated gas can be detected even though the cooling timescales can be considerably shorter than the outflow propagation timescales (Guillard et al. 2012), possibly thanks to the enhancement of turbulence on individualcloud scales (Nesvadba et al. 2011). Papadopoulos et al. (2008) and Ogle et al. (2010) previously attributed the overall strong

\footnotetext{
4 Heating of the molecular gas, leading to excitation at high rotational states, could be one of the reasons that absorption of the mm continuum by outflowing molecules is not (reliably) detected in $\mathrm{CO}(0-1)$ in the $\mathrm{PdB}$ data, while it was detected in $\mathrm{CO}(2-3)$ in the single-dish data. Alternatively, absorption in $\mathrm{CO}\left(0_{-1}\right)$ may not be seen if it is diluted in $\mathrm{CO}(1-0)$ emission. A similar scenario may not hold for $\mathrm{CO}(2-3)$ and $\mathrm{CO}(3-2)$ depending, e.g., on the size and the geometry of the emitting regions. Changes with time in the relative position of the foreground clouds and the background source leading to changes in the covering factor, or changes with time in the millimeter continuum flux could also justify this result.
} 
emission by $\mathrm{CO}$ molecules in states of intermediate rotational number and by $\mathrm{H}_{2}$ molecules in states of low rotational number to shock excitation by radio jets.

\subsection{Impact of the outflow on the galaxy}

The outflow could affect the future evolution of $4 \mathrm{C} 12.50$ via gas heating or gas expulsion. While the effects of heating are uncertain, we observe that the feedback mechanism has not had enough time to reach and excite a large fraction of the $\mathrm{CO}$ reservoir.

To test the role of gas expulsion, we computed the mass outflow rate, $\dot{M}_{\text {out }}$, which is equal to $M_{\text {out }} V_{\text {out }} d^{-1}$. We revised the flow rate of warm gas presented in Dasyra \& Combes (2011) from $130 M_{\odot} \mathrm{yr}^{-1}$ to $230 M_{\odot} \mathrm{yr}^{-1}$, using identical values for the outflow velocity $V_{\text {out }}\left(640 \mathrm{~km} \mathrm{~s}^{-1}\right)$ and mass $M_{\text {out }}$ $\left(5.2 \times 10^{7} M_{\odot}\right)$, but assuming that the entrained gas is within a distance $d$ of $150 \mathrm{pc}$ from the radio core (Morganti et al. $2013 \mathrm{~b})$. The flow of cold gas could carry up to $570 M_{\odot} \mathrm{yr}^{-1}$, under the assumption that the entrained $\mathrm{CO}$ and the entrained $\mathrm{H}_{2}$ have the same average velocity. This assumption does not conflict with the $\mathrm{CO}(2-3)$ detection at $-950 \mathrm{~km} \mathrm{~s}^{-1}$ (Dasyra $\&$ Combes 2012), which only probes the motions of some outflowing clouds: those situated between us and the radio core. For both gas phases, the total mass flow rate is in the range $230-800 M_{\odot} \mathrm{yr}^{-1}$.

However, only a part of this gas could be lost to the intergalactic medium. The escape velocity at $150 \mathrm{pc}$ from the western nucleus of $4 \mathrm{C} 12.50$ is $700 \mathrm{~km} \mathrm{~s}^{-1}$, when assuming an isothermal sphere distribution for the visible baryonic matter and when using a stellar velocity dispersion of $170 \mathrm{~km} \mathrm{~s}^{-1}$ and a half-light radius of $2.6 \mathrm{kpc}$ (Dasyra et al. 2006a). The escape velocity will increase to $800 \mathrm{~km} \mathrm{~s}^{-1}$ when the mass in the eastern nucleus of $4 \mathrm{C} 12.50$ is accounted for. From the $\mathrm{H}_{2}$ velocity distribution in the Spitzer data, less than $30 \%$ of the gas could be lost to the intergalactic medium. Most of the accelerated and heated gas will fountain back to a disk within a few dynamical timescales. The maximum mass loss rate would be $\lesssim 240 M_{\odot} /$ yr, and the minimum depletion timescale of the reservoir $\gtrsim 4 \times 10^{7} \mathrm{yrs}$. The depletion timescale could increase further and exceed the typical values for AGN duty cycles when the mass in the dark matter halo(s) is taken into account.

Any gas expulsion could be moderated or counteracted by a potential inflow of gas from the southern tidal tail. At the projected distance of the tail, $\sim 10 \mathrm{kpc}$, the escape velocity from the visible baryonic matter is in the range $200-300 \mathrm{~km} \mathrm{~s}^{-1}$, i.e., higher than the typical rotational velocities of spiral arms. Given that the line-of-sight velocity of the tidal tail is $<40 \mathrm{~km} \mathrm{~s}^{-1}$, gas could be captured and fed to the western nucleus.

We thus postulate that both gas expulsion and heating are likely to temporarily delay but unlikely to entirely suppress star formation in $4 \mathrm{C} 12.50$.

\subsection{Driving mechanism of the outflow}

Power and momentum arguments are typically invoked for evaluating the outflow driver. The flow kinetic luminosity can be computed from the product $0.5 M_{\text {out }} V_{\text {out }}^{3} d^{-1}$. For the $M_{\text {out }}, V_{\text {out }}$, and $d$ values discussed in Sect. 4.2, the kinetic luminosity of the warm entrained gas that is probed by the $\mathrm{H}_{2}$ is $3 \times 10^{43} \mathrm{erg} \mathrm{s}^{-1}$. Adding the maximum amount of cold outflowing gas that could be probed by the $\mathrm{CO}$ would place the kinetic luminosity upper limit to $1 \times 10^{44} \mathrm{erg} \mathrm{s}^{-1}$. The luminosity radiated by the

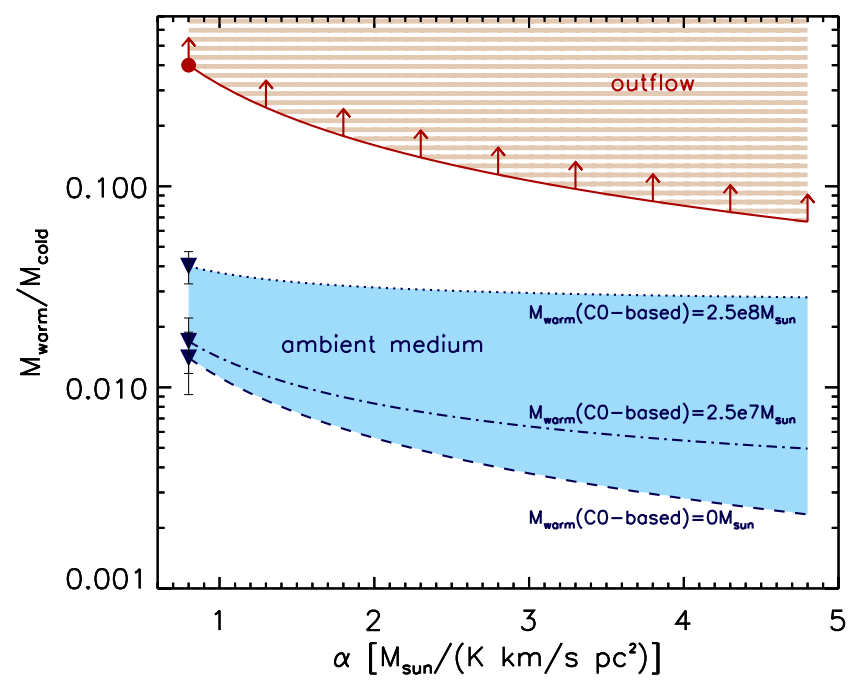

Fig. 13. Warm-to-cold gas mass ratio as a function of the $\alpha$ conversion factor. The circle shows the ratio's lower limit in the outflow for $\alpha=0.8 M_{\odot} /\left(\mathrm{K} \mathrm{km} \mathrm{s}^{-1} \mathrm{pc}^{2}\right)$. The solid line shows how this lower limit decreases as $\alpha$ approaches values thought appropriate for the Milky Way. This curve brackets the range of all acceptable $M_{\text {warm }} / M_{\text {cold }}$ values in the outflow (hatched area), under the assumption that the $400 \mathrm{~K}$ outflowing gas fraction probed by the $\mathrm{CO}$ is negligible compared to the fraction probed by the $\mathrm{H}_{2}$. For the ambient medium, the measured ratio (triangles) and its dependence on $\alpha$ (non-solid lines) are given for different warm gas content scenarios: when only the mass of the $400 \mathrm{~K} \mathrm{H}_{2}$ gas directly seen by Spitzer is used (dashed line) and when the mass of another CO-based $400 \mathrm{~K}$ gas component is added. The dashed-dotted line uses an additional mass of $2.5 \times 10^{7} M_{\odot}$ distributed in clouds of $2 \times 10^{6} \mathrm{~cm}^{-3}$ (as in the dashed-dotted CO SLED component in Fig. 11). The dotted line instead uses an additional mass of $2.5 \times 10^{8} M_{\odot}$ distributed in clouds of $1 \times 10^{5} \mathrm{~cm}^{-3}$ (as in the dotted CO SLED component in Fig. 11). This line constrains the upper range of acceptable $M_{\text {warm }} / M_{\text {cold }}$ values in the ambient medium (solid area).

outflowing gas in the purely rotational $\mathrm{H}_{2}$ lines, $4 \times 10^{41} \mathrm{erg} \mathrm{s}^{-1}$ (Dasyra \& Combes 2011), is negligible with respect to the outflow kinetic luminosity. The luminosity radiated in $\mathrm{CO}$ lines is even lower than that radiated in $\mathrm{H}_{2}$ lines.

According to Veilleux et al. (2009), half of the infrared luminosity of this source can be ascribed to its AGN and half to its starburst, with an uncertainty of $\sim 50 \%$ on either fraction. The dust-based star-formation-rate estimate is then $400 M_{\odot} / \mathrm{yr}$ (Kennicutt 1998). The power released by supernovae (SN) is $10^{44} \mathrm{erg} \mathrm{s}^{-1}$, under the assumption that for every hundred solar masses forming there is one SN (e.g., in the Kroupa 2001, initial mass function context), ejecting material with a kinetic energy of $10^{51} \mathrm{erg}$. This energy is insufficient to drive the flow unless a very large fraction $(30-100 \%)$ of it is converted into the outflow kinetic luminosity.

The luminosity of the AGN, like that of the starburst, is estimated to be $5 \times 10^{45} \mathrm{erg} \mathrm{s}^{-1}$. The force exerted to the gas by radiation pressure is then 5 to 18 times lower than the outflow momentum rate, when the latter is approximated by the product $\dot{M}_{\text {out }} V_{\text {out }}$ (Combes et al. 2013). The momentum rate boost needed for the gas acceleration could occur with the aid of energy-conserving gas expansion or multiple photon scatterings in high optical depth regions (Faucher-Giguère \& Quataert 2012). For these mechanisms, the compact-source geometry of the AGN is advantageous.

The AGN jet constitutes a generous power source, capable of driving the flow. The radio cavity kinetic luminosity is estimated 
from its $178 \mathrm{MHz}$ flux to be $3 \times 10^{45} \mathrm{erg} \mathrm{s}^{-1}$ (Guillard et al. 2012). The most significant argument in favor of the radio jet remains the location of the H I outflow at the tip of the southern radio hot spot (Morganti et al. 2013b). The young age of the jet, limited to $<10^{5}$ yrs (Lister et al. 2003), could explain why only a small amount of the $\mathrm{CO}$ is affected by the feedback.

\subsection{Implications for future observations}

Recent hydrodynamic simulations indicate that gas heating could be common in AGN outflows: the effects of jets and radiation-driven ultra-fast winds on the ISM can be comparable only $10^{4}-10^{5}$ years after the onset of the feedback activity (Wagner \& Bicknell 2011; Wagner et al. 2013), when both mechanisms drive bubbles of similar flow structure and dynamics.

If heating of the accelerated molecular gas is common, then the detection of outflows could be easier in intermediate$J$ CO lines than in low- $J$ CO lines, facilitating the discovery of outflows in the distant Universe: at $z \sim 1$ or higher, for example, ALMA could probe outflows of comparable mass to those presently detected at $100 \mathrm{Mpc}$ with the PdB. Moreover, the acquisition of infrared data alongside millimeter data could be valuable for the systematic discovery and reliable mass determination of outflows. With ALMA operating in full-array mode in about two years and with the James Webb Space Telescope coming up beyond 2018, these goals will be achievable for most systems of interest in the local Universe. At $100 \mathrm{Mpc}$, ALMA is designed to detect $5 \times 10^{5} \mathrm{M}_{\odot}$ outflows of $10 \mathrm{~K}$ gas in $\mathrm{CO}(1-0)$. The JWST is designed to reach the same limit for the $\sim 100 \mathrm{~K}$ gas probed by $\mathrm{H}_{2}(0-0) \mathrm{S}(1)$. For typical outflow radial extents of a few hundred pc, this common detection threshold corresponds to an outflow rate of $\sim 1 M_{\odot} \mathrm{yr}^{-1}$, comparable to the star formation rate in local spirals.

\section{Conclusions}

We used IRAM PdB, $30 \mathrm{~m}$ telescope, and Herschel Space Telescope data to study the content, excitation, and kinematic properties of the molecular gas in the outflow and in the ambient medium of a local prototype radio-loud, ultraluminousinfrared galaxy, 4C12.50 (also known as IRAS 13451+1232). We specifically looked for gas heating and expulsion signatures, i.e., characteristics of the two feedback-related processes that can delay star formation. Combining new and previous observations, we found evidence that both processes are taking place, and we drew the following main conclusions.

- The CO(1-0) emission spatially consists of a newly discovered tidal tail, which extends out to $12 \mathrm{kpc}$ southwest of the nucleus, and a main component, which is marginally resolved within the beam radius $(4.2 \mathrm{kpc})$. A shift in the location of the maximum emission with velocity indicates that the main component could be tracing either two merging nuclei or a rotating disk. Its reservoir contains $1.0( \pm 0.1) \times$ $10^{10} M_{\odot}$ of cold $\mathrm{H}_{2}$ gas for $\alpha=0.8 M_{\odot} /\left(\mathrm{K} \mathrm{km} \mathrm{s}^{-1} \mathrm{pc}^{2}\right)$.

- The CO SLED, which is primarily tracing the ambient gas in $4 \mathrm{C} 12.50$, is very similar with that of the Milky Way gas for low- $J$ numbers. Fitting of the CO SLED with RADEX indicates that the gas mass probed by $\mathrm{CO}$ molecules at $400 \mathrm{~K}$ is less than 0.025 times the gas mass probed by $\mathrm{CO}$ molecules at $25 \mathrm{~K}$. Likewise, the $\mathrm{H}_{2}$ gas mass probed by $\mathrm{CO}$ molecules at $400 \mathrm{~K}$ is less than 1.7 times the mass of the $400 \mathrm{~K}$ gas probed by the purely rotational $\mathrm{H}_{2}$ lines in the mid-infrared.
- In the outflow, the mass of the cold $(25 \mathrm{~K}) \mathrm{H}_{2}$ gas is $<1.3( \pm 0.1) \times 10^{8} M_{\odot}$, i.e., at most twice as high as the mass of the warm $(400 \mathrm{~K}) \mathrm{H}_{2}$ gas emitting in the mid-infrared. By combining the $\mathrm{H}_{2}$-based mass flow rate measurement from the mid-infrared data, $230 M_{\odot} / \mathrm{yr}$, with the CO-based mass flow rate upper limit from the mm data, $570 M_{\odot} / \mathrm{yr}$, we conclude that the feedback mechanism(s) could be accelerating up to $800 M_{\odot} / \mathrm{yr}$ of molecular gas for an outflow radius of $150 \mathrm{pc}$.

- A small fraction of the outflowing gas $(<30 \%$ when ignoring dark matter) could escape the system and be lost to the intergalactic medium. Any mass loss could be moderated or counteracted by a potential inflow of gas from the tidal tail containing $3.3( \pm 0.3) \times 10^{8} M_{\odot}$ of cold $\mathrm{H}_{2}$. The star formation in the host of $4 \mathrm{C} 12.50$ is likely to be delayed, but not entirely suppressed by the feedback.

- The mass ratio of warm-to-cold $\mathrm{H}_{2}$ gas is elevated in the outflow with respect to the ambient medium by a factor of $\gtrsim 30$ for $\alpha=0.8 M_{\odot} /\left(\mathrm{K} \mathrm{km} \mathrm{s}^{-1} \mathrm{pc}^{2}\right)$. The conclusion that the accelerated molecular gas is heated is robust against two main sources of uncertainty: the chosen $\alpha$ conversion factor and the mass of the warm gas probed by the CO. While the discrepancy will be lower for higher values of either parameter, there is no combination of acceptable parameter values that could make the warm-to-cold gas mass ratio in the ambient medium reach the ratio in the outflow.

- A scenario in which outflows could be probed by a different CO SLED than the rest of the ISM is likely. It remains to be tested with submillimeter observations.

Acknowledgements. K.M.D. thanks F. Casoli, O. La Marle, and M. Lozach for making this project possible by means of a French Space Agency (Centre National d'Études Spatiales; CNES) fellowship. Our work is based on data obtained with the facilities of IRAM, which is supported by INSU/CNRS (France), MPG (Germany), and IGN (Spain), and on data obtained with the Herschel Space Telescope, which is an ESA space observatory with science instruments provided by European-led principal investigator consortia and with important participation from NASA.

\section{References}

Aalto, S., Garcia-Burillo, S., Muller, S., et al. 2012, A\&A, 537, A44 Alatalo, K., Blitz, L., Young, L. M., et al. 2011, ApJ, 735, 88 Batcheldor, D., Tadhunter, C., Holt, J., et al. 2007, ApJ, 661, 70 Bernardi, M., Meert, A., Sheth, R. K., et al. 2013, MNRAS, 436, 697 Birnboim, Y., Dekel, A., \& Neistein, E. 2007, MNRAS, 380, 339 Booth, C. M., \& Schaye, J. 2009, MNRAS, 398, 53

Bower, R. G., Benson, A. J., Malbon, R., et al. 2006, MNRAS, 370, 645 Cantalupo, S., Lilly, S. J., \& Haehnelt, M. G. 2012, MNRAS, 425, 1992 Carter, M., Lazareff, B., Maier, D., et al. 2012, A\&A, 538, A89

Cicone, C., Feruglio, C., Maiolino, R., et al. 2012, A\&A, 543, A99 Cicone, C., Maiolino, R., Sturm, E., et al. 2014, A\&A, 562, A21 Combes, F., Garcia-Burillo, S., Casasola, V., et al. 2013, A\&A, 558, A124

Clements, D. L., Dunne, L, \& Eales, S. 2010, MNRAS, 403, 274 Croft, S., van Breugel, W., de Vries, W., et al. 2006, ApJ, 647, 1040 Croton, D. J., Springel, V., White, S. D. M., et al., 2006, MNRAS, 367, 864 Dasyra, K. M., \& Combes, F. 2011, A\&A, 533, L10 Dasyra, K. M., \& Combes, F. 2012, A\&A, 541, L7

Dasyra, K. M., Tacconi, L. J., Davies, R. I., et al. 2006a, ApJ, 638, 745 Dasyra, K. M., Tacconi, L. J., Davies, R. I., et al. 2006b, ApJ, 651, 835 Debuhr, J., Quataert, E., \& Ma, C.-P. 2012, MNRAS, 420, 2221

Dekel, A., \& Birnboim, Y. 2008, MNRAS, 383, 119

Downes, D., \& Solomon, P. M. 1998, ApJ, 507, 615

Draine, B. T. 2003, ARA\&A, 41, 241

Eckart, A., Cameron, M., Genzel, R., et al. 1990, ApJ, 365, 522 Fabian, A. C. 2012, ARA\&A, 50, 455

Faucher-Giguère, C.-A., \& Quataert, E. 2012, MNRAS, 425, 605 Feruglio, C., Maiolino, R., Piconcelli, E., et al. 2010, A\&A, 518. L155 Fischer, J., Sturm, E., González-Alfonso, E., et al. 2010, A\&A, 518, L41 Fu, H., \& Stockton, A. 2009, ApJ, 690, 953

Gaibler, V., Khochfar, S., Krause, M., \& Silk, J. 2012, MNRAS, 425, 438 
K. M. Dasyra et al.: Heating of the molecular gas in the massive outflow of 4C12.50

Golombek, D., Miley, G. K., \& Neugebauer, G. 1988, AJ, 95, 26

Granato, G. L., De Zotti, G., Silva, L., Bressan, A., \& Danese, L. 2004, ApJ, 600,580

Griffin, M. J., Abergel, A., Abreu, A., et al. 2010, A\&A, 518, L3

Guillard, P., Ogle, P., Emonts, B., et al. 2012, ApJ, 747, 95

Hailey-Dunsheath, S., Sturm, E., Fischer, J., et al. 2012, ApJ, 755, 57

Heckman, T. M., Lehnert, M. D., Strickland, D. K., \& Armus, L. 2000, ApJS, 129,493

Holt, J., Tadhunter, C. N., \& Morganti, R. 2008, MNRAS, 387, 639

Holt, J., Tadhunter, C. N., Morganti, R., \& Emonts, B. H. C. 2011, MNRAS, 410,1527

Ivison, R. J., Smail, I., Amblard, A., et al. 2012, MNRAS, 425, 1320

Kennicutt, R. C. 1998, ApJ, 498, 541

Krichbaum, T. P., Alef, W., Witzel, A., et al. 1998, A\&A, 329, 873

Krichbaum, T. P., Graham, D., Witzel, A., et al. 2001, ASP Conf., 250, 184

Krichbaum, T. P., Lee, S. S., Lobanov, A. P., et al. 2008, ASP Conf., 386, 186

Krips, M., Martín, S., Eckart, A., et al. 2011, ApJ, 736, 37

Kroupa, P. 2001, MNRAS, 322, 231

Leon, S., Eckart, A., Laine, S., et al. 2007, A\&A, 473, 747

Lípari, S., Mediavilla, E., Diaz, R. J., et al. 2004, MNRAS, 348, 369

Lister, M. L., Kellermann, K. I., Vermeulen, R. C., et al. 2003, ApJ, 584, L135

Maloney, P., \& Black, J. H. 1988, ApJ, 325, 389

Martin, C. L. 2005, ApJ, 621, 227

Meijerink, R., Kristensen, L. E., Weiss, A., et al. 2013, ApJ, 762, L16

Morganti, R., Tadhunter, C. N., \& Oosterloo, T. A. 2005, A\&A, 444, L9

Morganti, R., Frieswijk, W., Oonk, R. J. B., Oosterloo, T., \& Tadhunter, C. 2013a, A\&A, 552, L4

Morganti, R., Fogasy, J., Paragi, Z., et al. 2013b, Science, 341, 1082

Moshir, M., Kopan, G., Conrow, T., et al. 1990, BAAS, 22, 1325

Nesvadba, N., Boulanger, F., Lehnert, M., et al. 2011, A\&A, 536, L5

Ogle, P., Boulanger, F., \& Guillard, P. 2010, ApJ, 724, 1193

Ostorero, L., Moderski, R., Stawarz, L., et al. 2010, ApJ, 715, 1071

Ott, S. 2010, ASP Conf. Ser., 434, 139

Papadopoulos, P. P., Kovacs, A., Evans, A. S., \& Barthel, P. 2008, A\&A, 491, 483
Papadopoulos, P. P., van der Werf, P., Xilouris, E., Isaak, K., \& Gao, Y. 2012, ApJ, 751, 10

Pilbratt, G. L., Riedinger, J. R., Passvogel, T., et al. 2010, A\&A, 518, L1

Poglitsch, A., Waelkens, C., Geis, N., et al. 2010, A\&A, 518, L2

Rangwala, N., Maloney, P. R., \& Glenn, J. 2011, ApJ, 743, 94

Rupke, D. S., Veilleux, S., \& Sanders, D. B. 2005, ApJ, 632, 751

Rupke, D. S. N., \& Veilleux, S. 2011, ApJ, 729, L27

Sajina, A., Yan, L., Lutz, D., et al. 2008, ApJ, 683, 659

Sakamoto, K., Ho, P. T. P., \& Peck, A. B. 2006, ApJ, 644, 862

Sakamoto, K., Aalto, S., \& Wilner, D. J. 2009, ApJ, 700, L104

Sanders, D. B., \& Mirabel, I. F. 1996, ARA\&A, 34, 749

Silk, J. 2013, ApJ, 772, 112

Solomon, P. M., Downes, D., Radford, S. J. E., \& Barrett, J. W. 1997, ApJ, 478, 144

Somerville, R. S., Hopkins, P. F., Cox, T. J., et al. 2008, MNRAS, 391, 481 Spinoglio, L., Pereira-Santaella, M., Busquet, G., et al. 2012, ApJ, 758, 108

Spoon, H. W. W., Farrah, D., Lebouteiller, V., et al. 2013, ApJ, 775, 127

Steppe, H., Jeyakumar, S., Saikia, D. J., \& Salter, C. J. 1995, A\&AS, 113, 409

Sturm, E., González-Alfonso, E., Veilleux, S., et al. 2011, ApJ, 733, L16

Trippe, S., Neri, R., Krips, M., et al. 2010, A\&A, 515, A40

Tsai, A.-L., Matsushita, S., Kong, A. K. H., et al. 2012, ApJ, 752, 38

van Breugel, W., Filippenko, A. V., Heckman, T., \& Miley, G. 1985, ApJ, 293. 83

van der Tak, F. F. S., Black, J. H., Schöier, F. L., Jansen, D. J., \& van Dishoeck, E. F. 2007, A\&A, 468, 627

Veilleux, S., Rupke, D., Kim, D. C., et al. 2009, ApJS, 182, 628

Veilleux, S., Meléndez, M., Sturm, E., et al. 2013, ApJ, 776, 27

Wagner, A. Y., \& Bicknell, G. V. 2011, ApJ, 728, 29

Wagner, A. Y., Umemura, M., \& Bicknell, G. V. 2013, ApJ, 763, L18

Weiss, A., Neininger, N., Hüttemeister, S., \& Klein, U. 2001, A\&A, 365, 571

Weiss, A., Walter, F., \& Scoville, N. Z. 2005, A\&A, 438, 533

Westmoquette, M. S., Clements, D. L., Bendo, G. J., \& Khan, S. A. 2012, MNRAS, 424, 416

Wiklind, T., \& Combes, F. 1997, A\&A, 324, 51 